\title{
Higher order corrections to adiabatic invariants of generalized slow-fast Hamiltonian systems
}

\author{
M. Avendaño-Camacho, 1,a) J. A. Vallejo, , b) and Yu. Vorobiev, c) \\ Departamento de Matemáticas, Universidad de Sonora (México)
}

(Dated: 29 May 2022)

\begin{abstract}
We present a coordinate-free approach for constructing approximate first integrals of generalized slow-fast Hamiltonian systems, based on the global averaging method on parameter-dependent phase spaces with $\mathbb{S}^{1}$-symmetry. Explicit global formulas for approximate second-order first integrals are derived. As examples, we analyze the case quadratic in the fast variables (in particular, the elastic pendulum), and the charged particle in a slowly-varying magnetic field.
\end{abstract}

PACS numbers: 45.10.Na,45.10.Hj,02.30.Mv,02.30.Ik

Keywords: Adiabatic invariants, normal forms, slow-fast systems.

\footnotetext{
a) Electronic mail: misaelave@mat.uson.mx

b)Electronic mail: jvallejo@fc.uaslp.mx; In sabbatical leave. Permanent address: Facultad de Ciencias, UASLP (México)

c)Electronic mail: yurimv@guaymas.uson.mx
} 


\section{INTRODUCTION}

Slow-fast Hamiltonian systems appear in the theory of adiabatic approximation ${ }^{3,13}, 18,21,24,26$. They are represented by equations of motion of the form

$$
\begin{gathered}
\dot{y}=-\frac{\partial H}{\partial x}, \quad \dot{x}=\frac{\partial H}{\partial y} \\
\dot{p}=-\varepsilon \frac{\partial H}{\partial q}, \quad \dot{q}=\varepsilon \frac{\partial H}{\partial p}
\end{gathered}
$$

where $\varepsilon$ is a small perturbation parameter, and $(y, x) \in \mathbb{R}^{2 r},(p, q) \in \mathbb{R}^{2 k}$ are said to be fast and slow variables, respectively. This system is Hamiltonian relative to the function $H=H(p, q, y, x)$ and the rescaled canonical Poisson bracket on the product space $\mathbb{R}^{2 r} \times \mathbb{R}^{2 k}$ :

$$
\{f, g\}=\left(\frac{\partial f}{\partial y} \frac{\partial g}{\partial x}-\frac{\partial f}{\partial x} \frac{\partial g}{\partial y}\right)+\varepsilon\left(\frac{\partial f}{\partial p} \frac{\partial g}{\partial q}-\frac{\partial f}{\partial q} \frac{\partial g}{\partial p}\right)
$$

Notice that the corresponding symplectic form

$$
\sigma=d y \wedge d x+\frac{1}{\varepsilon} d p \wedge d q
$$

has a singularity at $\varepsilon=0$.

Usually, systems like (I.1) appear as a result of applying a scaling argument to an $\varepsilon$-dependent Hamiltonian on the standard phase space $\left(\mathbb{R}^{2 r+2 k}, d Y \wedge d X+d P \wedge d Q\right)$. Two common situations arise:

(1) Slowly varying Hamiltonians. In this case the original Hamiltonian has the form

$$
H\left(\varepsilon^{k} P, \varepsilon^{1-k} Q, Y, X\right), \quad(0 \leq k \leq 1),
$$

and the rescaling is implemented through the equations

$$
p=\varepsilon^{k} P, \quad q=\varepsilon^{1-k} Q, \quad y=Y, \quad x=X,
$$

which lead to

$$
H\left(\varepsilon^{k} P, \varepsilon^{1-k} Q, Y, X\right)=H(p, q, y, x) .
$$

(2) Rapidly varying Hamiltonians. Now the original Hamiltonian is

$$
H\left(P, Q, \frac{Y}{\varepsilon^{k}}, \frac{X}{\varepsilon^{1-k}}\right), \quad(0 \leq k \leq 1),
$$


Adiabatic invariants of slow-fast systems

and the rescaling

$$
p=P, \quad q=Q, \quad y=\frac{Y}{\varepsilon^{k}}, \quad x=\frac{X}{\varepsilon^{1-k}},
$$

leads to another one in the new variables:

$$
H(P, Q, Y, X)=\varepsilon H(p, q, y, x) .
$$

In both cases, the dynamics is described by (I.1)

These standard adiabatic models can be generalized in the following way $20,28,29$. Suppose we start with two symplectic manifolds $\left(M_{0}, \sigma_{0}\right)$ and $\left(M_{1}, \sigma_{1}\right)$. Consider the product manifold $M=M_{0} \times M_{1}$ endowed with canonical projections $\pi_{0}: M \rightarrow M_{0}$ and $\pi_{1}: M \rightarrow M_{1}$, and an $\varepsilon$-dependent symplectic form

$$
\sigma=\pi_{0}^{*} \sigma_{0}+\frac{1}{\varepsilon} \pi_{1}^{*} \sigma_{1}
$$

for $\varepsilon \neq 0$. The Poisson bracket determined by $\sigma$ is

$$
\{,\}=\{,\}_{0}+\varepsilon\{,\}_{1},
$$

where $\{f, g\}_{0}=\Psi_{0}(d f, d g)$ and $\{f, g\}_{1}=\Psi_{1}(d f, d g)$ are the Poisson brackets on $M$ defined as the canonical lifts of the non-degenerate Poisson brackets on the symplectic factors $\left(M_{0}, \sigma_{0}\right)$ and $\left(M_{1}, \sigma_{1}\right)$, respectively. The Poisson bivector fields $\Psi_{0}$ and $\Psi_{1}$ on $M$ are degenerate, as $\operatorname{rank} \Psi_{0}=\operatorname{dim} M_{0}$, and $\operatorname{rank} \Psi_{1}=\operatorname{dim} M_{1}$, and the corresponding symplectic leaves are given by the slices $M_{0} \times\left\{m_{1}\right\}$ and $\left\{m_{0}\right\} \times\left\{M_{1}\right\}$. If $(p, q, y, x)$ denote a local coordinate system on $M$ associated to the Darboux coordinates $(p, q)$ on $\left(M_{1}, \sigma_{1}\right)$ and $(y, x)$ on $\left(M_{0}, \sigma_{0}\right)$, then:

$$
\Psi_{0}=\frac{\partial}{\partial y} \wedge \frac{\partial}{\partial x}, \text { and } \Psi_{1}=\frac{\partial}{\partial p} \wedge \frac{\partial}{\partial q}
$$

The Poisson brackets $\{,\}_{0}$ and $\{,\}_{1}$ will be called fast and slow, respectively.

From the viewpoint of deformation theory, the adiabatic-type Poisson bracket (I.3) is nontrivial in the following sense. The bivector field determined by (1.3) reads $\Psi=\Psi_{0}+$

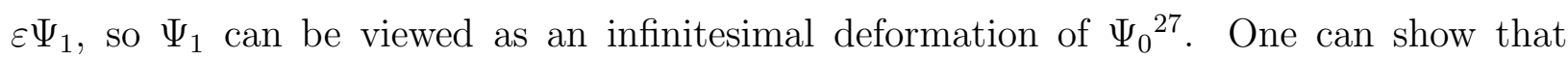
this deformation is nontrivial, that is, the Poisson cohomology class of the 2-cocycle $\Psi_{1}$ is nonzero.

For every function $H \in C^{\infty}(M)$, denote by $X_{H}$ the Hamiltonian vector field of $H$ relative to $\sigma, \mathbf{i}_{X_{H}} \sigma=-d H$. Then, $X_{H}=X_{H}^{(0)}+\varepsilon X_{H}^{(1)}$, where $X_{H}^{(0)}$ and $X_{H}^{(1)}$ are the Hamiltonian 
vector fields of $H$ with respect to $\{,\}_{0}$ and $\{,\}_{1}$, respectively: $X_{H}^{(0)}=\mathbf{i}_{d H} \Psi_{0}$ and $X_{H}^{(1)}=$ $\mathbf{i}_{d H} \Psi_{1}$. Also, denote by $d_{0}$ and $d_{1}$ the partial exterior derivatives on $M$ along $M_{0}$ and $M_{1}$, respectively. It is clear that $d=d_{0}+d_{1}$ is the exterior derivative on $M$ and $d_{0}^{2}=0=d_{1}^{2}$. Also, $d_{0} \circ d_{1}+d_{1} \circ d_{0}=0$. Then, the relations $X_{H}^{(0)}=\mathbf{i}_{d_{0} H} \Psi_{0}, X_{H}^{(1)}=\mathbf{i}_{d_{1} H} \Psi_{1}$ hold.

By a generalized slow-fast Hamiltonian system we mean a Hamiltonian system of the form:

$$
\left(M=M_{0} \times M_{1},\{,\}=\{,\}_{0}+\varepsilon\{,\}_{1}, H\right) .
$$

The corresponding Hamiltonian vector field $X_{H}=X_{H}^{(0)}+\varepsilon X_{H}^{(1)}$ gives rise to a perturbed dynamics, where the unperturbed vector field $X_{H}^{(0)}$ and the perturbation vector field $X_{H}^{(1)}$ are Hamiltonian with respect to different Poisson structures. As for any perturbed Hamiltonian system, it does make sense to search for adiabatic invariants, which are related to approximate first integrals of (I.4) as $\varepsilon \rightarrow 0$. We are interested in the existence of (additional to the Hamiltonian $H$ ) approximate first integrals of (I.4) for $\varepsilon \ll 1$, in the case when the flow of $X_{H}^{(0)}$ is periodic.

A $C^{\infty}$-function on $M$,

$$
F=F_{0}+\varepsilon F_{1}+\frac{\varepsilon^{2}}{2} F_{2}+\ldots+\frac{\varepsilon^{k}}{k !} F_{k}+O\left(\varepsilon^{k+1}\right),
$$

smoothly depending on the small parameter $\varepsilon \ll 1$, is said to be an approximate first integral of order $k \geq 0$ for $X_{H}=X_{H}^{(0)}+\varepsilon X_{H}^{(1)}$ if

$$
\mathcal{L}_{X_{H}} F=O\left(\varepsilon^{k+1}\right)
$$

An approximate first integral $F$ of order $k$ is an adiabatic invariant of order $k$ of the system (I.4), in the sense that

$$
\left|F \circ \mathrm{Fl}_{X_{H}}^{t}(p)-F(p)\right|=O\left(\varepsilon^{k}\right)
$$

for a long time scale: $t \sim \frac{1}{\varepsilon}$ as $\varepsilon \rightarrow 0$ (here $\mathrm{Fl}_{X_{H}}^{t}$ denotes the flow of the vector field $X_{H}$ ). For $k \geq 1$, the leading term $F_{0}$ of $F$ must be a common first integral of $X_{H}^{(0)}$ and the averaged perturbation vector field, and it is usually defined as the standard action (the classical adiabatic invariant) along the periodic trajectories of $X_{H}^{(0)} \underline{3}, 4,13$. Thus, the problem is to find the further corrections $F_{1}, F_{2}, \ldots$ Under appropriate hypotheses for the unperturbed (fast) dynamics, we show that a slow-fast Hamiltonian system (I.4) admits an approximate first integral of arbitrary order, and derive global coordinate-free formulas for the first and 
second order corrections $F_{1}$ and $F_{2}$. Our approach is based on the averaging method on general phase spaces with $\mathbb{S}^{1}$-symmetry $\underline{28,29}$ and results obtained elsewhere $\underline{16}, \underline{22,23}$. The main idea is to apply a global $\mathbb{S}^{1}$-normalization to (1.4) in two stages. The first step is related to the $\mathbb{S}^{1}$-averaging of the original $\varepsilon$-dependent symplectic form (the Poisson bracket) and then, in the second step, to apply the canonical normalization to the deformed Hamiltonian on the "new" phase space with $\mathbb{S}^{1}$-symmetry. This allows us to avoid the traditional assumption on the existence of action-angle variables, and to work on domains where the $\mathbb{S}^{1}$-action is not necessarily free and trivial. We illustrate these results with a two cases of physical interest: Hamiltonians quadratic in the fast variables (with the elastic pendulum as a particular example), and the charged particle in a slowly varying magnetic field.

The paper is organized as follows. In the next section we state our hypotheses and main result. Then, in section III we show how to construct the desired approximate first integrals when the perturbed system $X_{H}=X_{H}^{(0)}+\varepsilon X_{H}^{(1)}$ is in normal form relative to the $\mathbb{S}^{1}$-action on $M$ induced by $X_{H}^{(0)}$. To achieve this, a set of homological equations must be solved. In section [V] we prove that generalized slow-fast system satisfying our hypotheses also satisfy the conditions required to solve these homological equations. Section $\nabla$ is devoted to the proof of the main theorem, and the remaining sections analyze the examples.

\section{HIGHER ORDER CORRECTIONS TO ADIABATIC INVARIANTS}

Hypothesis 1 (Symmetry Hypothesis). We will assume that the flow of the unperturbed Hamiltonian vector field $X_{H}^{(0)}, \mathrm{Fl}_{X_{H}^{(0)}}$, is periodic on $M$ with frequency function $\omega \in C^{\infty}(M)$, $\omega>0$.

That means that $\mathrm{Fl}_{X_{H}^{(0)}}^{t+T(m)}(m)=\mathrm{Fl}_{X_{H}^{(0)}}^{t}(m)$ for all $t \in \mathbb{R}$ and $m \in M$. Here $T=\frac{2 \pi}{\omega}$ is the period function. Then, the flow of the vector field $\Upsilon:=\frac{1}{\omega} X_{H}^{(0)}$ is $2 \pi$-periodic and hence $\Upsilon$ is an infinitesimal generator of the $\mathbb{S}^{1}$-action on $M$. Let us associate ${ }^{5}$ to that $\mathbb{S}^{1}$-action the following operators acting on the space $\mathcal{T}_{l}^{s}(M)$ of all tensor fields on $M$ of type $(s, l)$ : the averaging operator $\langle\cdot\rangle: \mathcal{T}_{l}^{s}(M) \rightarrow \mathcal{T}_{l}^{s}(M)$,

$$
\langle A\rangle:=\frac{1}{2 \pi} \int_{0}^{2 \pi}\left(\mathrm{Fl}_{\Upsilon}^{t}\right)^{*} A d t
$$


and the integrating operator $\mathcal{S}: \mathcal{T}_{l}^{s}(M) \rightarrow \mathcal{T}_{l}^{s}(M)$,

$$
\mathcal{S}(A):=\frac{1}{2 \pi} \int_{0}^{2 \pi}(t-\pi)\left(\mathrm{Fl}_{\Upsilon}^{t}\right)^{*} A d t
$$

A tensor field $A \in \mathcal{T}_{l}^{s}(M)$ is said to be invariant with respect to the $\mathbb{S}^{1}$-action if $\left(\mathrm{Fl}_{\Upsilon}^{t}\right)^{*} A=A \quad($ for all $t \in \mathbb{R})$ or, equivalently, $\mathcal{L}_{\Upsilon} A=0$. In terms of the $\mathbb{S}^{1}$-average of $A$ the $\mathbb{S}^{1}$-invariance condition reads $A=\langle A\rangle$. In particular,

$$
\mathcal{L}_{\Upsilon}\langle A\rangle=0
$$

The integrating operator gives solutions to the following homological equation,

$$
\mathcal{L}_{\Upsilon} \circ \mathcal{S}(A)=A-\langle A\rangle
$$

It is clear that the frequency function $\omega$ and the Hamiltonian $H$ are $\mathbb{S}^{1}$-invariant. Moreover, by the period-energy relation $\underline{6,15}$ for periodic Hamiltonian flows, we have the equality

$$
d_{0} H \wedge d_{0} \omega=0
$$

This implies that the $\mathbb{S}^{1}$-action is canonical with respect to the fast bracket $\{,\}_{0}, \mathcal{L}_{\Upsilon} \Psi_{0}=0$. On the other hand, the $\mathbb{S}^{1}$-action does not preserve the slow Poisson bracket $\{,\}_{1}$, in general.

Property (II.3) says that the 1 -form $\frac{1}{\omega} d_{0} H$ is $d_{0}$-closed. Our next assumption strengthens this condition.

Hypothesis 2 (Parameterized Momentum Map). We assume that

$$
\frac{1}{\omega} d_{0} H \text { is } d_{0} \text {-exact }
$$

that is, there exists a smooth function $J: M \rightarrow \mathbb{R}$ such that

$$
\frac{1}{\omega} d_{0} H=d_{0} J
$$

and hence

$$
\Upsilon=\mathbf{i}_{d J} \Psi_{0}
$$

This means that the $\mathbb{S}^{1}$-action associated to the periodic flow of $X_{H}^{(0)}$ is Hamiltonian relative to $\{,\}_{0}$, with momentum map $J$. Also, notice that condition (II.4) holds whenever $M_{0}$ is simply connected. 
Adiabatic invariants of slow-fast systems

The momentum map $J$ is uniquely determined by (II.6) up to a transformation of the form

$$
J \mapsto J+c \circ \pi_{1}
$$

where $c$ is an arbitrary smooth function on $M_{1}$. Pick a $J$ and consider the 1 -form $d_{1} J$ on $M$ and its $\mathbb{S}^{1}$-average $\left\langle d_{1} J\right\rangle$.

Lemma II.1. There exists a closed 1 -form $\zeta \in \Omega^{1}\left(M_{1}\right)$ such that

$$
\left\langle d_{1} J\right\rangle=\pi_{1}^{*} \zeta
$$

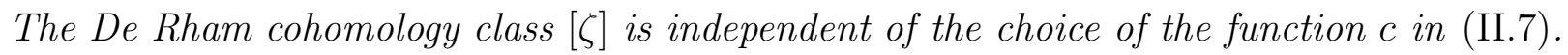

Hypothesis 3 (Adiabatic Condition ${ }^{22,23}$ ). We will assume that $[\zeta]=0$, and hence, there exists a momentum map $J \in C^{\infty}(M)$ in (II.6) satisfying the condition

$$
\left\langle d_{1} J\right\rangle=0
$$

Notice that the identities (II.5), (II.8), imply the relation

$$
\frac{1}{\omega} d_{1} H-d_{1} J=\frac{1}{\omega}\left\langle d_{1} H\right\rangle \text {. }
$$

From here and (ㅍ.5), we conclude that sufficient conditions for the differentials $d H$ and $d J$ to be linearly independent at a point $m \in M$, are $\left\langle d_{1} H\right\rangle_{m} \neq 0$ and $\left(d_{0} H\right)_{m} \neq 0$.

It is clear that condition (II.8) holds in the case when $M_{1}$ is simply connected.

Remark. ${ }^{22,23}$ If the "fast" symplectic manifold $\left(M_{0}, \sigma_{0}\right)$ is exact, that is, $\sigma_{0}=d \eta$ for some $\eta \in \Omega^{1}\left(M_{0}\right)$, then Hypothesis 1 implies Hypotheses 2 and [3. In this case, a momentum map $J$ satisfying the adiabatic condition (II.8) is given by the formula

$$
J=\frac{1}{\omega} \mathbf{i}_{X_{H}^{(0)}}\left\langle\pi_{0}^{*} \eta\right\rangle .
$$

In a domain where the $\mathbb{S}^{1}$-action is free, this formula gives the standard action ${ }^{3}$ along the periodic trajectories of $X_{H}^{(0)}$.

Example II.2. Let $M_{0}=\mathbb{S}^{2} \subset \mathbb{R}^{3}=\left\{\mathbf{x}=\left(x^{1}, x^{2}, x^{3}\right)\right\}$ is the unit sphere equipped with standard area form. Consider the Hamiltonian on $M=\mathbb{S}^{2} \times M_{1}$ of the form

$$
H=h+\omega \mathbf{n} \cdot \mathbf{x}
$$


Adiabatic invariants of slow-fast systems

for some smooth functions $h: M_{1} \rightarrow \mathbb{R}, \omega: M_{1} \rightarrow \mathbb{R}$ and $\mathbf{n}: M_{1} \rightarrow \mathbb{S}^{2}$. The corresponding fast Hamiltonian system satisfies all hypotheses above and the associated $\mathbb{S}^{1}$-action is given by the rotations in $\mathbb{R}_{\mathbf{x}}^{3}$ about the axis $-\mathbf{n}$. This action admits the momentum map $J=\mathbf{n} \cdot \mathbf{x}$, which satisfies (ㅍ.8).

Now, we state our main result.

Theorem II.3. Consider a slow-fast Hamiltonian system (I.4) and assume that the unperturbed (fast) Hamiltonian vector field $X_{H}^{(0)}$ satisfies the hypotheses 113 above. Let $J \in$ $C^{\infty}(M)$ be the momentum map of the associated $\mathbb{S}^{1}$-action, as in (II.6), satisfying the adiabatic condition (II.8). Then, for any $k \geq 0$, the perturbed Hamiltonian vector field $X_{H}=X_{H}^{(0)}+\varepsilon X_{H}^{(1)}$ admits an approximate first integral $F \in C^{\infty}(M)$, of order $k$, which is $\varepsilon$-close to $J$. In particular, for $k=2$, the formulae

$$
F=J+\varepsilon F_{1}+\frac{\varepsilon^{2}}{2} F_{2}
$$

where

$$
\begin{aligned}
F_{1}:=-\frac{1}{\omega} & \left(\mathcal{S}\left(\{H, J\}_{1}\right)+\frac{1}{2} \mathbf{i}_{d H}\left\langle\mathbf{i}_{\mathcal{S}\left(d_{1} J\right)} \Psi_{1}\right\rangle\right), \\
F_{2}: & =\frac{2}{\omega} \mathcal{S}\left(\left\{H, \frac{1}{\omega} \mathcal{S}\left(\{H, J\}_{1}\right)\right\}_{1}\right. \\
& \left.+\frac{1}{2}\left\{H, \mathbf{i}_{d H}\left\langle\mathbf{i}_{\mathcal{S}\left(d_{1} J\right)} \Psi_{1}\right\rangle\right\}_{1}\right)
\end{aligned}
$$

give an approximate first integral of order 2 ,

$$
\mathcal{L}_{X_{H}} F=O\left(\varepsilon^{3}\right)
$$

Let us remark once again that the expressions (ㅍ.10), (ㅍ.11), (II.12) are intrinsic (coordinate-free) and global. They involve just the $\mathbb{S}^{1}$-action induced by $X_{H}^{(0)}$, the Hamiltonian $H$, the "slow" Poisson bracket, and the averaging operators.

The following result follows from the remark above.

Corollary II.4. In the exact case, the assertions of Theorem II.3 remain true under just the symmetry hypothesis for $X_{H}^{(0)}$.

Also, from $\llbracket .3$ we get the following result $\underline{\underline{3} . \underline{4}}$.

Corollary II.5. If $\operatorname{dim} M_{0}=2=\operatorname{dim} M_{1},\left\langle d_{1} H\right\rangle \neq 0$ and $d_{0} H \neq 0$ on $M$, then, the 2-dimensional generalized slow-fast Hamiltonian system satisfying hypothesis 1 [ 3 is approximately integrable up to arbitrary order in $\varepsilon$. 


\section{APPROXIMATE FIRST INTEGRALS VIA NORMAL FORMS}

Suppose we are given, on a manifold $M$, a perturbed vector field of the form $A=A_{0}+\varepsilon A_{1}$. Then, a $C^{\infty}$-function on $M$,

$$
F=F_{0}+\varepsilon F_{1}+\frac{\varepsilon^{2}}{2} F_{2}+\ldots+\frac{\varepsilon^{k}}{k !} F_{k}
$$

is an approximate first integral of order $\varepsilon^{k}$ for $A$ if and only if the functions $F_{0}, \ldots, F_{k}$ are solutions to the following homological equations ${ }^{4}, 9$ :

$$
\begin{aligned}
\mathcal{L}_{A_{0}} F_{0} & =0, \\
\mathcal{L}_{A_{0}} F_{1} & =-\mathcal{L}_{A_{1}} F_{0}, \\
\mathcal{L}_{A_{0}} F_{2} & =-2 \mathcal{L}_{A_{1}} F_{1}, \\
& \ldots \\
\mathcal{L}_{A_{0}} F_{k} & =-k ! \mathcal{L}_{A_{1}} F_{k-1} .
\end{aligned}
$$

Assume that the flow of the unperturbed vector field $A_{0}$ is periodic with frequency function $\omega: M \rightarrow \mathbb{R}, \omega>0$, and consider the $\mathbb{S}^{1}$-action on $M$ with infinitesimal generator $\Upsilon:=\frac{1}{\omega} A_{0}$.

Proposition III.1. Assume that there exists a smooth function $J \in C^{\infty}(M)$ which is a common first integral of the unperturbed vector field $A_{0}$ and the averaged perturbation vector field $\left\langle A_{1}\right\rangle$, that is, $\mathcal{L}_{A_{0}} J=0$ and $\mathcal{L}_{\left\langle A_{1}\right\rangle} J=0$. Then:

(a) For an arbitrary $C_{1} \in C^{\infty}(M)$, the function

$$
F=J+\varepsilon\left(F_{1}^{0}-\left\langle C_{1}\right\rangle\right)
$$

where $F_{1}^{0}:=-\frac{1}{\omega} \mathcal{L}_{\mathcal{S}\left(A_{1}\right)} J$ satisfies $\left\langle F_{1}^{0}\right\rangle=0$, is an approximate second order first integral of $A$.

(b) If $C_{1}$ can be chosen so that the normalization condition of second order holds:

$$
\left\langle\mathcal{L}_{A_{1}} F_{1}^{0}\right\rangle=\left\langle\mathcal{L}_{\left\langle A_{1}\right\rangle} C_{1}\right\rangle
$$

then, $A$ admits an approximate first integral $F$, of order 2 , of the form

$$
F=J+\varepsilon\left(F_{1}^{0}-\left\langle C_{1}\right\rangle\right)+\frac{\varepsilon^{2}}{2}\left(F_{2}^{0}-\left\langle C_{2}\right\rangle\right)
$$


Adiabatic invariants of slow-fast systems

where

$$
F_{2}^{0}:=\frac{2}{\omega}\left(\mathcal{S} \circ \mathcal{L}_{A_{1}}\left(\frac{1}{\omega} \mathcal{L}_{\mathcal{S}\left(A_{1}\right)} J\right)-\mathcal{L}_{\mathcal{S}\left(A_{1}\right)}\left\langle C_{1}\right\rangle\right),
$$

satisfies $\left\langle F_{2}^{0}\right\rangle=0$, and $C_{2} \in C^{\infty}(M)$ is an arbitrary smooth function.

Proof. The statement follows from the solvability condition for homological equations (III.1) 5,9 .

Let us remark that the direct approach for finding approximate first integrals $\frac{13}{}$ leads to the verification of normalization condition (III.2), which is not necessarily satisfied in general. It is precisely here that our hypotheses come into play. As we will see, they will allow us first, to construct a normal form for the perturbed Hamiltonian vector field $X_{H}=X_{H}^{(0)}+\varepsilon X_{H}^{(1)}$, and then, from a first integral of the truncated normal form (which, in turn, is built out of the normalization transformation and the momentum map), an approximate first integral of the original system.

Proposition III.2. $\stackrel{5}{=}$ Under the symmetry hypothesis for $A_{0}$, for any $k>1$, the perturbed vector field $A=A_{0}+\varepsilon A_{1}$ admits an $\mathbb{S}^{1}$-invariant global normal form of order $k$,

$$
\mathcal{T}_{\varepsilon}^{*}\left(A_{0}+\varepsilon A_{1}\right)=A_{0}+\varepsilon\left\langle A_{1}\right\rangle+\frac{\varepsilon^{2}}{2} \bar{A}_{2}+\ldots+\frac{\varepsilon^{k}}{k !} \bar{A}_{k}+O\left(\varepsilon^{k+1}\right),
$$

where $\left\langle\bar{A}_{i}\right\rangle=\bar{A}_{i}$, for $i \in\{2, \ldots, k\}$.

The near-identity transformation $\mathcal{T}_{\varepsilon}$ is given by the time- $\varepsilon$ flow of an $\varepsilon$-dependent vector field on $M$. Therefore, the normalization transformation $\mathcal{T}_{\varepsilon}$ is well-defined on any relatively compact open subset in $M$ for small enough $\varepsilon$. The $\mathbb{S}^{1}$-invariant vector fields $\bar{A}_{2}, \ldots, \bar{A}_{k}$ are well-defined on the whole $M$, and they determine the truncated normal form ${ }^{\underline{5}}$ of order $k$ of A.

This leads to the following criterion.

Corollary III.3. Suppose that an $\mathbb{S}^{1}$-invariant smooth function $J: M \rightarrow \mathbb{R}$ is a first integral of the truncated $\mathbb{S}^{1}$-invariant normal form of order $k$ for $A$,

$$
\mathcal{L}_{\left\langle A_{1}\right\rangle} J=\mathcal{L}_{\bar{A}_{2}} J=\ldots=\mathcal{L}_{\bar{A}_{k}} J=0 .
$$

Then, the function

$$
F=J \circ \mathcal{T}_{\varepsilon}^{-1}=J+\varepsilon F_{1}+\frac{\varepsilon^{2}}{2} F_{2}+\ldots+\frac{\varepsilon^{k}}{k !} F_{k}+O\left(\varepsilon^{k+1}\right),
$$

where, for $s \in\{1, \ldots, k\}, F_{s}=\left.\frac{d^{s}}{d \varepsilon^{s}}\right|_{\varepsilon=0}\left(J \circ \mathcal{T}_{\varepsilon}^{-1}\right)$, is an approximate first integral on $M$ of order $k$ for $A$. 


\section{NORMALIZATION OF SLOW-FAST HAMILTONIAN SYSTEMS}

In this section, we show that conditions (III.3) are satisfied by generalized slow-fast Hamiltonian systems (I.4). To this end, we will assume that the unperturbed Hamiltonian vector field $X_{H}^{(0)}$ satisfies the hypotheses 1, 2, 3, We also suppose that a momentum map $J$ as in (II.6) is given, and that it satisfies the adiabatic condition (II.8). As mentioned above, generally the symplectic form $\sigma(\overline{\mathrm{I} .2})$ and the Poisson bracket (I.3) are not invariant with respect to the $\mathbb{S}^{1}$-action on $M$ associated to the periodic flow of $X_{H}^{(0)}$. This raises the question of the normalization of the perturbed Hamiltonian vector field $X_{H}=X_{H}^{(0)}+\varepsilon X_{H}^{(1)}$ relative to the $\mathbb{S}^{1}$-action. To get $\mathbb{S}^{1}$-invariant global normal forms, one can apply to $X_{H}$ a non-canonical (non-Hamiltonian) Lie transform $\operatorname{method}^{5}$. But our point is to maintain the Hamiltonian setting. For that purpose, we proceed the normalization procedure in two steps. In the first stage, we correct the drawbacks of our $\varepsilon$-dependent phase space by constructing an $\mathbb{S}^{1}$-invariant Poisson bracket $\{,\}^{\text {inv }}$ (a symplectic form) which is $\varepsilon$-close to the original one $\{$,$\} . In a second stage, we normalize the deformed Hamiltonian H \circ \Phi_{\varepsilon}$ up to desired order in $\varepsilon$ by applying a near-identity transformation which will be defined as the Hamiltonian flow relative to $\{,\}^{\text {inv }}$. Here $\Phi_{\varepsilon}$ is a Poisson isomorphism between $\{,\}^{\text {inv }}$ and $\{$,$\} . Thus, this second step is basically a modification of the Deprit algorithm 11$ for $\varepsilon$-dependent phase spaces.

Let us associate to the momentum map $J$ the 1 -form (on $M) \Theta:=\mathcal{S}\left(d_{1} J\right)$, which has the property $\langle\Theta\rangle=0$.

Lemma IV.1. The average $\langle\sigma\rangle$ of the symplectic form (1.2) has the representation $\langle\sigma\rangle=$ $\sigma-d \Theta$. Moreover, for any $\mathbb{S}^{1}$-invariant, relatively compact $N \subset M$, and small enough $\varepsilon$, the averaged 2-form $\langle\sigma\rangle$ is non-degenerate on $N$, and there exists a near-identity transformation $\Phi_{\varepsilon}: N \rightarrow M,\left(\Phi_{0}=\mathrm{id}\right)$ which is a symplectomorphism between $\langle\sigma\rangle$ and $\sigma, \Phi_{\varepsilon}^{*} \sigma=\langle\sigma\rangle$.

Proof. The proof of this statement is based on a parametric version of the Moser homotopy method $10,17,28$, where the symplectomorphism $\Phi_{\varepsilon}$ is constructed as follows 10,28 . Fix an $\mathbb{S}^{1}$-invariant, relatively compact subset $N \subset M$. Then, define the family of 2 -forms on $M$ depending on the parameter $\lambda$

$$
\delta_{\lambda}:=d_{1} \Theta+\frac{(1-\lambda)}{2}\{\Theta \wedge \Theta\}_{0}
$$


Adiabatic invariants of slow-fast systems

where $\frac{1}{2}\{\Theta \wedge \Theta\}_{0}$ denotes the 2 -form defined by its action on a pair of vector fields $X, Y \in$ $\mathcal{X}(M)$ by

$$
\frac{1}{2}\{\Theta \wedge \Theta\}_{0}(X, Y):=\{\Theta(X), \Theta(Y)\}_{0},
$$

and consider the time-dependent vector field $Z_{\lambda}$ on $N$, depending on $\varepsilon$ as a parameter, and uniquely determined by the relations

$$
\begin{aligned}
\mathbf{i}_{Z_{\lambda}}\left(\pi_{1}^{*} \sigma_{1}-\varepsilon(1-\lambda) \delta_{\lambda}\right) & =-\Theta, \\
\mathbf{i}_{Z_{\lambda}} \pi_{0}^{*} \sigma_{0} & =0 .
\end{aligned}
$$

It follows that the flow of $\varepsilon Z_{\lambda}$ is well-defined on $N$, for small enough $\varepsilon$ and all $\lambda \in[0,1]$. Then, it suffices to take

$$
\Phi_{\varepsilon}=\left.\mathrm{Fl}_{\varepsilon Z_{\lambda}}^{\lambda}\right|_{\lambda=1}
$$

Now, denote by $\{f, g\}^{\text {inv }}=\Psi^{\text {inv }}(d f, d g)$ the non-degenerate Poisson bracket on $N$ associated to the averaged symplectic form $\langle\sigma\rangle$. Then, its Poisson bivector field $\Psi^{\mathrm{inv}}$ is $\mathbb{S}^{1}$-invariant, and it has the representation $\underline{28}-30$

$$
\Psi^{\mathrm{inv}}=\Psi_{0}+\varepsilon\left(\left\langle\Psi_{1}\right\rangle+\mathcal{L}\langle V\rangle \Psi_{0}\right)+O\left(\varepsilon^{2}\right)
$$

where

$$
V:=\frac{1}{2} \mathbf{i}_{\Theta} \Psi_{1}
$$

The following observation ${ }^{28}$ shows the role of adiabatic condition (II.8).

Lemma IV.2. Let $J$ be the momentum satisfying adiabatic condition (II.8). Then,

$$
\Upsilon=X_{J}^{(0)}=\mathbf{i}_{d J} \Psi^{\mathrm{inv}}
$$

that is, the $\mathbb{S}^{1}$-action is canonical on $\left(N,\{,\}^{\text {inv }}\right)$ with momentum map $J$.

Remark. The adiabatic condition (II.8) was introduced 22.23 in the context of the theory of Hannay-Berry connections on fibred phase spaces with symmetry. In our case, the 1-form $\Theta=\mathcal{S}\left(d_{1} J\right)$ appearing in formulas (II.11) and (II.12), just represents the Hamiltonian form of the Hannay-Berry connection on the trivial bundle $M_{0} \times M_{1} \rightarrow M_{1}$ over the "slow" base $M_{1}$ with the "fast" fiber $M_{0}$ endowed with an $\mathbb{S}^{1}$-action associated to the periodic Hamiltonian flow of $X_{H}^{(0)} \underline{16}, 28,29$. . If $\Theta$ is closed, then by Lemma IV.1] the original symplectic form (I.2) is $\mathbb{S}^{1}$-invariant. 
We arrive at the following normalization result.

Theorem IV.3. Suppose that the unperturbed Hamiltonian vector field $X_{H}^{(0)}$ satisfies the hypotheses 1, 2, 3. Then, for any $m \geq 1$ and small enough $\varepsilon$, there exists a near-identity transformation $\mathcal{T}_{\varepsilon}: N \rightarrow M$ which takes the original slow-fast Hamiltonian system (I.4) into an $\mathbb{S}^{1}$-invariant normal form of order $m$ of the form $\left(N,\{,\}^{\text {inv }}, H \circ \mathcal{T}_{\varepsilon}\right)$, where

$$
H \circ \mathcal{T}_{\varepsilon}=H+\sum_{s=1}^{m} \frac{\varepsilon^{s}}{s !}\left\langle K_{s}\right\rangle+O\left(\varepsilon^{m+1}\right),
$$

for some smooth functions $K_{1}, \ldots, K_{m}$ on $M$. In particular,

$$
K_{1}:=\frac{1}{2} \mathbf{i}_{d H} \mathbf{i}_{\Theta} \Psi_{1}
$$

Proof. In the first step, after applying the near-identity transformation $\Phi_{\varepsilon}(\mathrm{IV} .3)$ to the original system (I.4), we get a new perturbed Hamiltonian system relative to the $\mathbb{S}^{1}$-invariant Poisson bracket, $\left(N,\{,\}^{\text {inv }}, H \circ \Phi_{\varepsilon}\right)$, and a deformed Hamiltonian,

$$
H \circ \Phi_{\varepsilon}=H+\sum_{s=1}^{m} \frac{\varepsilon^{s}}{s !} \tilde{H}_{s}+O\left(\varepsilon^{m+1}\right) .
$$

Here the functions

$$
\tilde{H}_{s}=\left.\frac{d^{s}}{d \varepsilon^{s}}\right|_{\varepsilon=0}\left(H \circ \Phi_{\varepsilon}\right), \quad s \in\{1, \ldots, m\}
$$

are not necessarily $\mathbb{S}^{1}$-invariant. By applying Lemmas IV.1 and IV.2, a direct computation gives the result

$$
\tilde{H}_{1}=\frac{1}{2} \mathbf{i}_{d H} \mathbf{i}_{\Theta}\left(\left\langle\Psi_{1}\right\rangle+\mathcal{L}_{\langle V\rangle} \Psi_{0}+\Psi_{1}\right)
$$

where $K_{1}$ is given by (IV.8). In a second step, we normalize the truncated Taylor series (the $m$-th jet) of the deformed Hamiltonian (IV.9) by using the Hamiltonian flows relative to the $\mathbb{S}^{1}$-invariant Poisson bracket $\{,\}^{\text {inv }}$. Thus, we are looking for some smooth functions on $M, G_{0}, \ldots, G_{m-1}$, such that the time- $\varepsilon$ flow of the Hamiltonian vector field $\mathbf{i}_{d G} \Psi^{\text {inv }}$ of the function

$$
G=G_{0}+\sum_{i=1}^{m-1} \frac{\varepsilon^{i}}{i !} G_{i}
$$

gives us the desired normalization transformation,

$$
\left(H+\sum_{s=1}^{m} \frac{\varepsilon^{s}}{s !} \tilde{H}_{s}\right) \circ \mathrm{Fl}_{\mathbf{i}_{d G}^{\varepsilon} \Psi^{\mathrm{inv}}}=H+\sum_{s=1}^{m} \frac{\varepsilon^{s}}{s !}\left\langle K_{s}\right\rangle+O\left(\varepsilon^{m+1}\right) .
$$


By a standard Lie transform argument, we conclude that $G_{0}, \ldots, G_{m-1}$, must satisfy a set of homological equations on $M$ :

$$
\begin{gathered}
\left\{H, G_{0}\right\}_{0}=\mathcal{R}_{1}-\left\langle K_{1}\right\rangle, \\
\left\{H, G_{1}\right\}_{0}=\mathcal{R}_{2}-\left\langle K_{2}\right\rangle, \\
\cdots \\
\left\{H, G_{m-1}\right\}_{0}=\mathcal{R}_{m}-\left\langle K_{m}\right\rangle,
\end{gathered}
$$

where the functions $\mathcal{R}_{2}, \ldots, \mathcal{R}_{m}$ are defined by the recursive procedure given by the modified Deprit diagram. The corrections to the standard Deprit diagram 11 come from the Taylor expansion of the Poisson tensor $\Psi^{\text {inv }}$ in $\varepsilon$ at $\varepsilon=0$. In particular, by using (IV.4), (IV.5), one can show that

$$
\mathcal{R}_{1}=\tilde{H}_{1}
$$

and

$$
\begin{aligned}
\mathcal{R}_{2} & =\tilde{H}_{2}+\mathcal{L}_{\mathbf{i}_{d G_{0}} \Psi_{0}}^{2} H+2 \mathcal{L}_{\mathbf{i}_{d G_{0}} \Psi_{0}} \tilde{H}_{1} \\
& +\mathbf{i}_{d H} \mathbf{i}_{d G_{0}}\left(\left\langle\Psi_{1}\right\rangle+\mathcal{L}_{\langle V\rangle} \Psi_{0}\right) .
\end{aligned}
$$

To assure the solvability of the homological equations (IV.11), we choose the functions $K_{1}, \ldots, K_{m}$ in such a way that

$$
\left\langle\mathcal{R}_{1}\right\rangle=\left\langle K_{1}\right\rangle, \ldots,\left\langle\mathcal{R}_{m}\right\rangle=\left\langle K_{m}\right\rangle
$$

In particular, taking into account that $H$ and $\Psi_{0}$ are $\mathbb{S}^{1}$-invariant, and the property $\langle\Theta\rangle=0$, we deduce from (IV.10), ( $(\underline{\mathrm{IV} .12})$ and (IV.13), that $K_{1}$ is just given by (IV.8), and one can put

$$
K_{2}=\tilde{H}_{2}+\left\{\mathcal{S}\left(\frac{\tilde{H}_{1}}{\omega}\right), \tilde{H}_{1}\right\}_{0}
$$

The global solutions to the homological equations (IV.11) are given by the formulae $\mathrm{e}^{5}$,

$$
G_{0}=\frac{1}{\omega} \mathcal{S}\left(\mathcal{R}_{1}\right), \ldots, G_{m-1}=\frac{1}{\omega} \mathcal{S}\left(\mathcal{R}_{m}\right)
$$

Finally, the normalization transformation in (IV.7) is defined by

$$
\mathcal{T}_{\varepsilon}=\Phi_{\varepsilon} \circ \mathrm{Fl}_{\mathbf{i}_{d G} \Psi^{\mathrm{inv}}}^{\varepsilon}
$$


Corollary IV.4. Let $\mathcal{T}_{\varepsilon}$ be the normalization transformation in (IV.7) and $\tilde{H}=H \circ \mathcal{T}_{\varepsilon}$. Then, the momentum map $J$ is an approximate first integral of order $m$ for the Hamiltonian vector field $\mathbf{i}_{d \tilde{H}} \Psi^{\mathrm{inv}}$,

$$
\mathcal{L}_{\mathbf{i}_{d \tilde{H}} \Psi^{\text {inv }}} J=O\left(\varepsilon^{m+1}\right) .
$$

Proof. By using properties (IV.6) and (IV.7), we get

$$
\begin{aligned}
\mathcal{L}_{\mathbf{i}_{d \tilde{H}} \Psi^{\mathrm{inv}}} J & =\{\tilde{H}, J\}^{\mathrm{inv}}=\{\tilde{H}, J\}_{0}=-\mathcal{L}_{\Upsilon} \tilde{H} \\
& =-\mathcal{L}_{\Upsilon} H-\sum_{i=1}^{m} \frac{\varepsilon^{i}}{i !} \mathcal{L}_{\Upsilon}\left\langle K_{i}\right\rangle+O\left(\varepsilon^{m+1}\right) \\
& =O\left(\varepsilon^{m+1}\right)
\end{aligned}
$$

where, in the last equation, we have used the $\mathbb{S}^{1}$-invariance of $H$, and the property (II.1).

\section{PROOF OF THE MAIN RESULT}

Now, we have all the elements required to give a proof of Theorem II.3. Notice that

$$
X_{H}=\left(\mathcal{T}_{\varepsilon}^{-1}\right)^{*}\left(\mathbf{i}_{d \tilde{H}} \Psi^{\mathrm{inv}}\right)
$$

and hence by Corollary IV.4, the function $J \circ \mathcal{T}_{\varepsilon}^{-1}$ is an approximate first integral of order $m$ for $X_{H}$. This proves the first assertion of Theorem [I.3. In particular, by taking the Taylor expansion of second order

$$
J \circ \mathcal{T}_{\varepsilon}^{-1}=J+\varepsilon F_{1}+\frac{\varepsilon^{2}}{2} F_{2}+O\left(\varepsilon^{3}\right),
$$

we deduce from (III.1) that the functions $F_{1}$ and $F_{2}$ must satisfy the homological equations

$$
\mathcal{L}_{\Upsilon} F_{1}=-\frac{1}{\omega}\{H, J\}_{1}
$$

and

$$
\mathcal{L}_{\Upsilon} F_{2}=-\frac{1}{\omega}\left\{H, F_{1}\right\}_{1}
$$

A general solution to the first equation is given $b y^{\underline{5}}$

$$
F_{1}=-\frac{1}{\omega} \mathcal{S}\left(\{H, J\}_{1}\right)-\left\langle C_{1}\right\rangle
$$


for a function $C_{1} \in C^{\infty}(M)$ which has to satisfy the solvability condition for the second equation

$$
\frac{1}{\omega}\left\langle\left\{H, C_{1}\right\}_{1}\right\rangle=-\left\langle\left\{H, \frac{1}{\omega} \mathcal{S}\left(\{H, J\}_{1}\right\}_{1}\right\rangle .\right.
$$

It is difficult to find $C_{1}$ from this equation. Instead of following this approach, we will derive an explicit formula for $F_{1}$ by using the definition of $\mathcal{T}_{\varepsilon}$. Firstly, one can verify by a direct computation that the second term in the right-hand side of (V.1) is given by

$$
F_{1}=-\left\{G_{0}, J\right\}_{0}-\frac{1}{2} \mathbf{i}_{d J} \mathbf{i}_{\Theta} \Psi_{0}
$$

where $G_{0}$ is a solution to the homological equation (IV.11). From this fact, and properties (II.5), (II.9), we get the following result.

Lemma V.1. The second term in the Taylor expansion (V.1) is represented as follows

$$
F_{1}=-\frac{1}{\omega} \mathcal{S}\left(\{H, J\}_{1}\right)-\frac{1}{\omega}\left\langle K_{1}\right\rangle
$$

where $K_{1}$ is given by (IV.8).

Therefore, one can put $C_{1}=\frac{1}{\omega} K_{1}$. Finally, a particular solution to (V.1) is given by the formula $F_{2}=\frac{1}{\omega} \mathcal{S}\left(\left\{H, F_{1}\right\}_{1}\right)$, which leads to the representation (II.12). This ends the proof of Theorem II.3.

\section{HAMILTONIANS QUADRATIC IN THE FAST VARIABLES}

Let us particularize the previous developments in the case of a slow-fast Hamiltonian system $\left(\mathbb{R}_{(y, x)}^{2} \times \mathbb{R}_{(p, q)}^{2 k}, \frac{1}{\varepsilon} d p \wedge d q+d y \wedge d x, H\right)$ where the Hamiltonian $H$ is a quadratic function in the "fast" variables $\mathbf{z}=(y, x)$. To this end, let us associate to every matrixvalued function $\mathbf{A} \in \mathfrak{s l}(2, \mathbb{R}) \otimes C^{\infty}\left(\mathbb{R}^{2 k}\right)$ the function $Q_{\mathbf{A}}=-\frac{1}{2} \mathbf{J A z} \cdot \mathbf{z}$, where $\mathbf{J}=\left(\begin{array}{rr}0 & -1 \\ 1 & 0\end{array}\right)$, and the dot denotes the euclidian scalar product. The Hamiltonian vector field relative to the "fast" Poisson bracket $\{,\}_{0}$ is given by $X_{Q_{\mathbf{A}}}^{(0)}=\mathbf{A z} \cdot \frac{\partial}{\partial \mathbf{z}}$. Consider a Hamiltonian of the form $H=h+\omega Q_{\mathbf{A}}$, for some smooth functions $h=h(p, q)$ and $\omega=\omega(p, q)>0$. We assume that det $\mathbf{A}=1$ on an open domain in $\mathbb{R}_{(p, q)}^{2 k}$. This implies that $X_{H}^{(0)}$ has periodic flow with frequency function $\omega$, hence the infinitesimal generator of the $\mathbb{S}^{1}$-action is $X_{Q_{\mathbf{A}}}^{(0)}$, and the associated $\mathbb{S}^{1}$-action is given by the linear flow $\mathrm{Fl}_{\Upsilon}^{t}=\cos t \mathbf{I}+\sin t \mathbf{A}$. The corresponding momentum map is $J=Q_{\mathbf{A}}$. 
It is easy to see that hypotheses 13 hold in this case. For an arbitrary $\mathbf{S} \in \mathfrak{s l}(2, \mathbb{R}) \otimes$ $C^{\infty}\left(\mathbb{R}^{2 k}\right)$, we have the following identities,

$$
\begin{aligned}
\left\langle Q_{\mathbf{S}}\right\rangle & =\frac{1}{2} Q_{\mathbf{S}-\mathbf{A S A}}, \\
\mathcal{S}\left(Q_{\mathbf{S}}\right) & =\frac{1}{4} Q_{[\mathbf{A}, \mathbf{S}]} .
\end{aligned}
$$

The Hamiltonian vector field $X_{H}=X_{H}^{(0)}+\varepsilon X_{H}^{(1)}$ admits an approximate first integral $F$ of second order, $\mathcal{L}_{X_{H}} F=O\left(\varepsilon^{3}\right)$, of the form $F=J+\varepsilon F_{1}+\frac{\varepsilon^{2}}{2} F_{2}+O\left(\varepsilon^{3}\right)$, where $J$ is the momentum map and $F_{1}, F_{2}$ are given in (II.11), (II.12). In the quadratic case these formulae are reduced to:

$$
\begin{aligned}
F_{1}= & -\frac{1}{4 \omega}\left(Q_{[\mathbf{A}, \mathbf{B}]}+Q_{\mathbf{A}} Q_{[\mathbf{A}, \mathbf{C}]}\right) \\
& +\frac{\omega}{4} \sum_{i=1}^{k}\left(Q_{\mathbf{A} \frac{\partial \mathbf{A}}{\partial p^{i}}} Q_{\frac{\partial \mathbf{A}}{\partial q^{i}}}-Q_{\mathbf{A} \frac{\partial \mathbf{A}}{\partial q^{i}}} Q_{\frac{\partial \mathbf{A}}{\partial p^{i}}}\right), \\
= & -Q_{\frac{1}{4 \omega}\left([\mathbf{A}, \mathbf{B}]+Q_{\mathbf{A}}[\mathbf{A}, \mathbf{C}]\right)-\frac{\omega}{4} \widehat{Q}(\mathbf{A})},
\end{aligned}
$$

and,

$$
F_{2}=\frac{1}{2 \omega} Q_{\left[\mathbf{A},\left\{h, \frac{1}{4 \omega}\left([\mathbf{A}, \mathbf{B}]+Q_{\mathbf{A}}[\mathbf{A}, \mathbf{C}]\right)-\frac{\omega}{4} \widehat{Q}(\mathbf{A})\right\}_{1}\right]},
$$

where we have introduced the notations $\mathbf{B}:=\{h, \mathbf{A}\}_{1}, \mathbf{C}:=\{\omega, \mathbf{A}\}_{1}$ (the bracket being computed separately for each coefficient of the matrix A),

$$
\widehat{Q}(\mathbf{A}):=\sum_{i=1}^{k}\left(Q_{Q_{\mathbf{A} \frac{\partial \mathbf{A}}{\partial p^{i}}} \frac{\partial \mathbf{A}}{\partial q^{i}}}-Q_{\mathbf{A} \frac{\partial \mathbf{A}}{\partial q^{i}}} Q_{Q_{\mathbf{A} \frac{\partial \mathbf{A}}{\partial q^{i}}}} \frac{\partial \mathbf{A}}{\partial p^{i}}\right) .
$$

Example VI.1. We start from the Breitenberger-Mueller model for the Hamiltonian of the elastic pendulum ${ }^{8}$ :

$$
\tilde{H}=\frac{1}{2}\left(p_{x}^{2}+\omega_{p}^{2} x^{2}\right)+\frac{1}{2}\left(p_{y}^{2}+\omega_{s}^{2} y^{2}+\gamma x^{2} y\right)
$$

With the rescaling $x=\frac{\mathrm{X}}{\omega_{s}}, y=\omega_{p}^{2} \mathrm{Y}$, we get,

$$
\tilde{H}=\frac{1}{2}\left(p_{x}^{2}+\left(\frac{\omega_{p}}{\omega_{s}}\right)^{2} \mathrm{X}^{2}\right)+\frac{1}{2}\left(p_{y}^{2}+\left(\omega_{s} \omega_{p}^{2}\right)^{2} \mathrm{y}^{2}+\gamma\left(\frac{\omega_{p}}{\omega_{s}}\right)^{2} \mathrm{X}^{2} \mathrm{Y}\right)
$$

Next, we introduce the parameter $\varepsilon=\frac{\omega_{p}}{\omega_{s}}$, which according to the physical meaning of the problem, can be considered small: $\varepsilon \ll 1$. In terms of this parameter the Hamiltonian reads,

$$
\tilde{H}=\frac{1}{2}\left(p_{x}^{2}+\varepsilon^{2} \mathrm{X}^{2}\right)+\frac{1}{2}\left(p_{y}^{2}+\left(\omega_{s} \omega_{p}^{2}\right)^{2} \mathrm{y}^{2}+\gamma \varepsilon^{2} \mathrm{X}^{2} \mathrm{Y}\right)
$$


which can be written as $\tilde{H}=H\left(p_{x}, \varepsilon \mathrm{X}, p_{y}, \mathrm{Y}\right)$. A further rescaling $p_{x}=p, q=\varepsilon \mathrm{X}, y=p_{y}$ and $x=\mathrm{Y}$, leads to the slow-fast Hamiltonian system:

$$
H=\frac{1}{2}\left(p^{2}+q^{2}\right)+\frac{1}{2}\left(y^{2}+\Omega^{2} x^{2}+\gamma q^{2} x\right),
$$

where $\Omega=\omega_{s} \omega_{p}^{2}$, and the $\varepsilon$-dependent Poisson bracket $\{\}=,\{,\}_{0}+\varepsilon\{,\}_{1}$ on $\mathbb{R}^{2}(p, q) \times$ $\mathbb{R}^{2}(y, x)$ (both brackets, $\{,\}_{0}$ and $\{,\}_{1}$, are the canonical one on $\mathbb{R}^{2}$ ). The Hamiltonian vector field $X_{H}=X_{H}^{(0)}+\varepsilon X_{H}^{(1)}$ is readily computed,

$$
\begin{aligned}
& X_{H}^{(0)}=-\left(\Omega^{2} x+\frac{1}{2} \gamma q^{2}\right) \frac{\partial}{\partial y}+y \frac{\partial}{\partial x} \\
& X_{H}^{(1)}=-(1+\gamma x) q \frac{\partial}{\partial p}+p \frac{\partial}{\partial q} .
\end{aligned}
$$

The flow of $X_{H}^{(0)}$ is periodic with constant frequency function $\Omega$. The momentum map of the $\mathbb{S}^{1}$-action $J$ in given by

$$
J(p, q, y, x)=\frac{\Omega}{2}\left(x+\frac{\gamma}{2 \Omega^{2}} q^{2}\right)^{2}+\frac{1}{2 \Omega} y^{2}
$$

and, finally, the approximate first integral of second order for $X_{H}$ has the form

$$
\begin{aligned}
F & =J+\varepsilon \frac{\gamma}{\Omega^{3}} p q y \\
& +\varepsilon^{2} \frac{\gamma}{4 \Omega^{3}}\left(\gamma q^{2}\left(x+\frac{\gamma}{2 \Omega^{2}} q^{2}\right)\right. \\
& \left.\left(x-\frac{3 \gamma}{2 \Omega^{2}} q^{2}\right)+4\left(q^{2}-p^{2}\right)\left(x+\frac{\gamma}{2 \Omega^{2}} q^{2}\right)-\frac{\gamma}{\Omega^{2}} q^{2} y^{2}\right) .
\end{aligned}
$$

\section{CHARGED PARTICLE IN A SLOWLY VARYING MAGNETIC FIELD}

The adiabatic invariants appearing in the motion of charged particles in a slowly varying magnetic field are well-known in plasma physics since long ago $\stackrel{1,7,14,21}{ }$, they are related to physical phenomena such as magnetic traps ${ }^{3}$. Indeed, for this problem explicit expressions for corrections of the classical first and second adiabatic invariants have been given up to first order $\underline{12}, 19,25$. Here, we will consider a cylindrically symmetric configuration for the magnetic field (in cylindrical coordinates)

$$
\mathbf{B}=B_{r} \mathbf{u}_{r}+B_{z} \mathbf{u}_{z}=B \mathbf{u}_{r}-B \frac{z}{r} \mathbf{u}_{z}
$$


Notice that $\frac{\partial B_{r}}{\partial r}=0$, so, rather than magnetic traps, we will study a force-free contracting plasmoid.

Let us write the equations of motion of a charged $(e=1)$ particle in this field in such a way that the slow and fast components are apparent ${ }^{13}$. In terms of the Clebsch potentials

$$
\left\{\begin{array}{l}
\alpha(\varepsilon r, \theta, \varepsilon z)=-B \varepsilon^{2} r z, \\
\beta(\varepsilon r, \theta, \varepsilon z)=\theta
\end{array}\right.
$$

we have $\mathbf{B}=\frac{1}{\varepsilon^{2}} \boldsymbol{\nabla} \alpha \times \boldsymbol{\nabla} \beta$; also, we can write $\mathbf{B}=\nabla \times \mathbf{A}$, where $\mathbf{A}=\frac{1}{\varepsilon^{2}} \alpha \nabla \beta$. The Hamiltonian is given by $H=\frac{1}{2}\|\widetilde{\mathbf{p}}-\mathbf{A}\|^{2}$, where $\widetilde{\mathbf{p}}$ is the kinematical momentum. Let us apply the canonical transformation with generating function

$$
F=\frac{S}{\varepsilon} p_{2}+\frac{\beta}{\varepsilon} p_{1}+\frac{\alpha}{\varepsilon} p_{3}-p_{1} p_{3}
$$

where we have introduced another variable, $S$, such that $\frac{S}{\varepsilon}$ is the arc-length along along field lines so, in the far-field regime $r \gg 1$ (which will be the one of interest for us)

$$
\frac{S}{\varepsilon} \simeq r
$$

Notice that this transformation, being canonical, does not alter the (canonical) Poisson brackets. From (VII.2), we get

$$
\left\{\begin{array}{l}
\beta=\varepsilon q_{1}+\varepsilon q_{3} \\
S=\varepsilon q_{2} \\
\alpha=\varepsilon q_{3}+\varepsilon p_{1}
\end{array} .\right.
$$

Now, $\widetilde{\mathbf{p}}=\nabla F=\frac{p_{2}}{\varepsilon} \nabla S+\frac{p_{1}}{\varepsilon} \nabla \beta+\frac{p_{3}}{\varepsilon} \nabla \alpha$. So $\widetilde{\mathbf{p}}-\mathbf{A}=\frac{p_{2}}{\varepsilon} \nabla S+\frac{p_{1}}{\varepsilon} \nabla \beta+\frac{p_{3}}{\varepsilon} \nabla \alpha-\frac{1}{\varepsilon^{2}} \alpha \nabla \beta$. Substituting (VII.4):

$$
\widetilde{\mathbf{p}}-\mathbf{A}=\frac{p_{2}}{\varepsilon} \nabla S+\frac{p_{3}}{\varepsilon} \nabla \alpha-\frac{q_{3}}{\varepsilon} \nabla \beta
$$

Thus

$$
\|\widetilde{\mathbf{p}}-\mathbf{A}\|^{2}=p_{2}^{2}+\varepsilon^{2} B^{2} p_{3}^{2}\left(r^{2}+z^{2}\right)+\frac{q_{3}^{2}}{\varepsilon^{2} r^{2}}-2 \varepsilon B z p_{2} p_{3} .
$$

Now, we expand this expression in powers of $\left(p_{3}, q_{3}\right)$; to this end, we use $\alpha=\varepsilon p_{1}, \beta=\varepsilon q_{1}$, $S=\varepsilon q_{2}$, which, together with (VII.1), (VII.3), gives $z=-\frac{p_{1}}{\varepsilon B q_{2}}$ and $r=q_{2}$. Thus, we get a Hamiltonian explicitly displaying slow and fast variables:

$$
\begin{aligned}
& H\left(q_{1}, \varepsilon p_{1}, \varepsilon q_{2}, p_{2}, q_{3}, p_{3}\right)= \\
& \frac{1}{2}\left[p_{2}^{2}+p_{3}^{2}\left(B^{2}\left(\varepsilon q_{2}\right)^{2}+\frac{\left(\varepsilon p_{1}\right)^{2}}{\left(\varepsilon q_{2}\right)^{2}}\right)+2 \frac{\left(\varepsilon p_{1}\right) p_{2}}{\left(\varepsilon q_{2}\right)} p_{3}+\frac{q_{3}^{2}}{\left(\varepsilon q_{2}\right)^{2}}\right] .
\end{aligned}
$$


After the obvious rescaling $\widetilde{p}_{1}=\varepsilon p 1, \widetilde{q}_{2}=\varepsilon q_{2}$, dropping the tildes for simplicity, and noticing that $q_{1}$ is a cyclic variable (so we can take $p_{1}=\lambda$, a parameter) we obtain the Hamiltonian system

$$
\begin{aligned}
& H\left(q_{2}, p_{2}, q_{3}, p_{3}\right)= \\
& \frac{1}{2}\left[p_{2}^{2}+p_{3}^{2}\left(B^{2} q_{2}^{2}+\frac{\lambda^{2}}{q_{2}^{2}}\right)+2 \lambda \frac{p_{2}}{q_{2}} p_{3}+\frac{q_{3}^{2}}{q_{2}^{2}}\right] .
\end{aligned}
$$

The unperturbed Hamiltonian vector field ("fast" variables) is given by

$$
X_{H}^{(0)}=-\frac{q_{3}}{q_{2}} \frac{\partial}{\partial p_{3}}+\left(\frac{B^{2} q_{2}^{4}+\lambda^{2}}{q_{2}^{2}} p_{3}+\lambda \frac{p_{2}}{q_{2}}\right) \frac{\partial}{\partial q_{3}},
$$

and it has periodic flow with frequency function

$$
\omega=\frac{\sqrt{B^{2} q_{2}^{4}+\lambda^{2}}}{q_{2}^{2}}>0 .
$$

The flow is of the infinitesimal generator of the $\mathbb{S}^{1}$-action $\Upsilon=\frac{1}{\omega} X_{H}^{(0)}$ is given by

$$
\mathrm{Fl}_{\Upsilon}^{t}\left(\begin{array}{c}
p_{2} \\
q_{2} \\
p_{3} \\
q_{3}
\end{array}\right)=\left(\begin{array}{c}
p_{2} \\
q_{2} \\
\left(p_{3}+\lambda \frac{p_{2}}{\omega^{2} q_{2}^{3}}\right) \cos (t)-\frac{q_{3}}{\omega q_{2}^{2}} \sin (t)-\lambda \frac{p_{2}}{\omega^{2} q_{2}^{3}} \\
\left(\omega q_{2}^{2} p_{3}+\lambda \frac{p_{2}}{\omega q_{2}}\right) \sin (t)+q_{3} \cos (t)
\end{array}\right),
$$

and the momentum map reads

$$
J\left(p_{2}, q_{2}, p_{3}, q_{3}\right):=\frac{1}{2 \omega}\left(\frac{q_{3}^{2}}{q_{2}^{2}}+\left(\omega q_{2} p_{3}+\lambda \frac{p_{2}}{\omega q_{2}^{2}}\right)^{2}\right) .
$$

A straightforward computation shows that, indeed, $J=\frac{v_{\perp}^{2}}{B}$, where $v_{\perp}$ is the transverse momentum. From our previous results, we see that the Hamiltonian vector field $X_{H}=$ $X_{H}^{(0)}+\varepsilon X_{H}^{(1)}$ admits an approximate first integral $F$ to any arbitrary order in $\varepsilon$. In particular, to second order it has the form $F=J+\varepsilon J_{1}+\frac{\varepsilon^{2}}{2} J_{2}+O\left(\varepsilon^{3}\right)$, where $J$ is the momentum map, and

$$
J_{1}\left(p_{2}, q_{2}, p_{3}, q_{3}\right)=-\frac{q_{3}}{q_{2}^{10} \omega^{5}}\left(p_{2} p_{3} q_{2}^{9} B^{4}+\lambda q_{2}^{4}\left(\lambda^{2} p_{3}^{2}-2 p_{2}^{2} q_{2}^{2}\right) B^{2}+\lambda^{3}\left(q_{3}^{2}+\left(p_{2} q_{2}+\lambda p_{3}\right)^{2}\right)\right) .
$$

The explicit expression for $J_{2}$ is too long to be displayed here (it can be easily computed with the aid of a computer algebra system such as Maxima). 
Adiabatic invariants of slow-fast systems

\section{REFERENCES}

${ }^{1}$ H. Alfvén, Cosmical Electrodynamics, Clarendon Press, Oxford, 1950.

${ }^{2}$ V. I. Arnold, Mathematical Methods of Classical Mechanics, Springer Verlag, New York, 1978.

${ }^{3}$ V. I. Arnold, Small denominators and problems of stability of motion in classical and celestial mechanics, Russ. Math. Surveys, 186 (1963) 85-191.

${ }^{4}$ V. I. Arnold, V. V. Kozlov and A. I. Neishtad, Mathematical aspects of classical and celestial mechanics (Dynamical Systems III), 3rd Ed., Springer Verlag, Berlin, 2006.

${ }^{5} \mathrm{M}$. Avendaño-Camacho and Yu. Vorobiev, Homological equations for tensor fields and periodic averaging, Russ. J. Math. Phys. 183 (2011), 243-257.

${ }^{6}$ L. Bates and J. Sniatycki, On the period-energy relation, Proc. of Amer. Math. 1143 (1992) 877-878.

${ }^{7}$ M. Braun, Particle motions in a magnetic field, J. of Diff. Eq. 8 (1970) 294-332.

${ }^{8}$ E. Breitenberger and R. D. Mueller, The elastic pendulum: A nonlinear paradigm, J. Math. Phys. 226 (1981) 1196-1211.

${ }^{9}$ R. Cushman, Normal form for Hamiltonian vector fields with periodic flow. In: "Differential Geometric Methods in Mathematical Physics" (S. Sternberg, ed.), Reidel, Boston, 1984, pp. 125-144.

${ }^{10} \mathrm{G}$. Dávila-Rascón and Yu. Vorobiev. A Hamiltonian approach for skew-product dynamical systems, Russian J. of Math. Phys. 151 (2008), 35-44.

${ }^{11}$ A. Deprit, Canonical transformation depending on a small parameter, Cel. Mech. 72 (1969) $173-179$.

${ }^{12} \mathrm{R}$. Gall, Motion of charged particles in slowly varying fields to the first order of approximation, J. of Geophys. Res. 6812 (1963) 3565-3577.

${ }^{13}$ C. S. Gardner, Adiabatic invariants of periodic classical systems, Phys. Rev. 1154 (1959) 791-795.

${ }^{14}$ L. M. Garrido and F. Gascón, Approximate constancy of adiabatic invariants in plasma physics, Prog. of Theor. Phys. 284 (1962) 573-585.

${ }^{15} \mathrm{~W}$. Gordon, On the relation between period and energy in periodic dynamical systems, J. Math. Mech. 19 (1969) 111-114.

${ }^{16}$ S. Golin, A. Knauf and S. Marmi, The Hannay angles: geometry, adiabaticity, and an 
Adiabatic invariants of slow-fast systems

example, Comm. Math. Phys. 123 (1989) 95-122.

${ }^{17}$ V. Guillemin, E. Lerman, and S. Sternberg, Symplectic Fibrations and Multiplicity Diagrams, Cambridge Univ. Press, Cambridge, 1996.

${ }^{18} \mathrm{M}$. V. Karasev, New global asymptotics and anomalies for the problem of quantization of the adiabatic invariant, Funct. Anal. Appl. 242 (1990) 104-114.

${ }^{19}$ M. Kruskal, The gyration of a charged particle, Princeton University report PM-S-33, NYO-7903 (1958).

${ }^{20}$ E. A. Kudryavtseva, Periodic solutions of planetary systems with satellites and the averaging method in systems with slow and fast variables, arXiv:1201.6356v6.

${ }^{21}$ R. G. Littlejohn and S. Weigert, Adiabatic motion of a neutral spinning particle in an inhomogeneous magnetic field, Phys. Rev. A 48 (1993) 924-940.

${ }^{22}$ J. E. Marsden, R. Montgomery and T. Ratiu, Reduction, symmetry and phases in mechanics, Mem. of the AMS, 88436 (1990) 1-110.

${ }^{23} \mathrm{R}$. Montgomery, The connection whose holonomy is the classical adiabatic angles of Hannay and Berry and its generalization to the non-integrable case, Comm. Math. Phys. 120 (1988) 269-294.

${ }^{24}$ A. Neishtadt, Averaging method and adiabatic invariants. In: "Hamiltonian dynamical systems and applications" (W. Craig ed.), Springer Verlag, 2008, 53-66.

${ }^{25}$ T. G. Northrop, C. S. Liu and M. D. Kruskal, First correction to the second adiabatic invariant of charged-particle motion, Phys. Fluids 9 (1966) 1503-1515.

${ }^{26}$ J. A. Sanders, F. Verhulst and J. Murdock, Averaging methods in nonlinear dynamical systems, Springer Verlag, New York, 2007.

${ }^{27}$ I. Vaisman, Lectures on the geometry of Poisson manifolds, Prog. in Math. 118, Birkhauser, Boston, 1994.

${ }^{28} \mathrm{Yu}$. Vorobiev, The averaging in Hamiltonian systems on slow-fast phase spaces with $\mathbb{S}^{1}$ symmetry, Phys. of Atom. Nucl. 747 (2011), 1-5.

${ }^{29} \mathrm{Yu}$. Vorobiev and M. Avendaño-Camacho, The averaging method on slow-fast phase spaces with symmetry, J. Phys.: Conf. Ser. 343 (2012) 1-11.

${ }^{30} \mathrm{Yu}$. Vorobiev and M. Avendaño-Camacho, On the global structure of normal forms for slow-fast Hamiltonian systems, to appear at Russ. J. of Math. Phys. 202 (2013). 